[7] U-X. Tan, W. T. Latt, C. Y. Shee, and W. T. Ang, "Design and development of a low-cost flexure-based hand-held micromanipulator," in Proc. IEEE Intl. Conf. Robot. Autom., Kobe, Japan, May 2009, pp. 4350 4355.

[8] D. Y. Choi and C. N. Riviere, "Flexure-based manipulator for active handheld microsurgical instrument," in Proc. 27th Annu. Intl. Conf. IEEE Eng. Medicine Biol. Soc. (EMBS), Shanghai, China, Sep. 2005, pp. 2325 2328.

[9] S Henein, S. Bottinelli, and R. Clavel, "Parallel spring stages with flexures of micrometric cross-sections," in Proc. SPIE Microrobotics Microsys. Fabrication, Pittsburgh, PA, 1998, pp. 209-220.

[10] X. Y. Tang and I.-M. Chen, "A large-displacement 3-DOF flexure parallel mechanism with decoupled kinematics structure," in Proc. IEEE/RS J Intl. Conf. Intell. Robots Syst., Beijing, China, Oct. 2006, pp. 16681673.

[11] Objet. (2008). [Online]. Available: www.objet.com/Pages/FullCure_ Materials_Data_Sheets/Vero_Family

[12] I. A. Bonev, "Direct kinematics of zero-torsion parallel mechanisms," in Proc. IEEE Intl. Conf. Robot. Autom., Pasadena, CA, 2008, pp. 38513856.

[13] Les Prototypes. (2009). [Online]. Available: www-sop.inria. frmembersJean-Pierre.MerletPhotosection3_2.html

[14] J. G. Avedillo, D. Choi, and C. N. Riviere, "Inverse kinematic model of flexure-based microsurgical manipulator," IEEE Intl. Conf. Eng. Medicine Biol. Soc., Sep. 2005, pp. 5775-5777.

[15] K. M. Lee and D. K. Shah, "Kinematic analysis of a three-degrees of freedom in-parallel actuated mechanism," IEEE J. Robot. Autom., vol. 4, no. 3, pp. 354-360, Jun. 1988

[16] K. M. Lee and D. K. Shah, "Dynamic analysis of a three-degrees-offreedom in-parallel actuated manipulator," IEEE J. Robot. Autom., vol. 4 no. 3, pp. 361-367, Jun. 1988.

[17] K. M. Lee and S. Arjunan, "A three-degrees-of-freedom micromotion inparallel actuated manipulator," in Proc. IEEE Trans. Robot. Autom., Oct., 1991, vol. 7, no. 5, pp. 634-641.

[18] W. T. Ang, C. N. Riviere, and P. K. Khosla, "Feedforward controller with inverse rate-dependent model for piezoelectric actuators in trajectorytracking applications," IEEE/ASME Trans. Mechatronics, vol. 12, no. 2, pp. 1-8, Apr. 2007.

[19] U-X. Tan, W. T. Latt, C. Y. Shee, C. N. Riviere, and W. T. Ang, "Feedforward controller of ill-conditioned hysteresis using singularity-free Prandtl-Ishlinskii model," IEEE/ASME Trans. Mechatronics, vol. 14, no. 5, pp. 598-605, Oct. 2009.

[20] U-X. Tan, W. T. Latt, F. Widjaja, C. Y. Shee, C. N. Riviere, and W. T. Ang, "Tracking control of hysteretic piezoelectric actuator using adaptive ratedependent controller," Sens. Actuators A, Phys., vol. 150, no. 1, pp. 116$123,2009$.

[21] T. L. Win, U. X. Tan, C. Y. Shee, and W. T. Ang, "Design and calibration of an optical micro motion sensing system for micromanipulation tasks," in Proc. IEEE Intl. Conf. Robot. Autom., Roma, Italy, Apr. 2007, pp. 33833388 .

\section{An Integrated Triangulation Laser Scanner for Obstacle Detection of Miniature Mobile Robots in Indoor Environment}

\author{
Guoqiang Fu, Paolo Corradi, Arianna Menciassi, \\ and Paolo Dario
}

\begin{abstract}
The miniaturization of an autonomous robot requires the integration of components that not only need to satisfy strict spatial constraints, but also need to demonstrate useful functionalities and performance, while demanding low power. For miniaturized autonomous robots that aim at exploring unknown environments, sensors for navigation and for the understanding of basic geometrical features of the environment are of utmost importance for a robot's survival and mission. This paper presents a miniaturized triangulation laser scanner that was developed and characterized for use on a $10 \times 10 \times 10 \mathrm{~cm}^{3}$ robot. The optimal configurations of the laser sensor on two sides of the robot are discussed, and measurement formulas as well as theoretical resolution are deduced. For indoor applications, the measurement range of the system runs from approximately $80 \mathrm{~mm}$, with $1 \mathrm{~mm}$ resolution, up to $600 \mathrm{~mm}$, with $12 \mathrm{~mm}$ resolution. The aim of the work is to demonstrate the possibility of extracting basic information from the robot surroundings by means of small, simple, low-power, and low-cost demanding devices, which, in addition, can be scaled down in order to equip even smaller robots.
\end{abstract}

Index Terms-Indoor navigation, laser scanner, miniature mobile robot, triangulation.

\section{INTRODUCTION}

In order to perform safe, noncontact exploration of uncertain environments, autonomous mobile robots often employ range sensors to support navigation, mapping, and localization tasks. To estimate the distance to objects, these sensors often use ultrasonic [1], infrared [2], or laser techniques [3], [4]. Vision capability based on the use of cameras may also be employed [5]-[7], yet this technique requires the support of higher processing capabilities, especially in nonstructured environments. A major drawback of ultrasonic and IR sensors is the low spatial resolution in regard to both distance detection and object scanning. Laser-based range sensors can offer a highly focused structured light, which can be used for a more refined and computationally simpler understanding of the unknown environment by simply elaborating the structured-light pattern, as acquired by an integrated camera. In addition, structured light allows the robot to quite accurately measure the geometric features of surrounding objects, thus contributing to the decision-making process of the robot when it accomplishes tasks, or more simply for safer navigation and exploration.

Two kinds of laser range scanners may be used for measuring and scanning applications: time-of-flight (TOF) or phase-shift laser range scanners and triangulation laser range scanners. Due to the small size of the target robots [8] (which was determined by a tradeoff between miniaturization, on-board power, and actuation capability), low power availability, and the need to maintain low cost for each robot (in order to

Manuscript received February 14, 2010; revised May 31, 2010 and August 27, 2010; accepted August 28, 2010. Date of publication November 9, 2010; date of current version May 11, 2011. Recommended by Technical Editor C. A. Kitts. This work was supported by the EU FP7 "Cognitive Systems, Interaction, Robotics" large-scale integrating project "Robotic Evolutionary Self-Programming and Self-Assembling Organisms-REPLICATOR" under Grant 216240.

The authors are with the Center for Research in Microengineering, Scuola Superiore Sant'Anna, 56025 Pontedera, Italy (e-mail: g.fu@ @ssup.it; paolo.corradi@sssup.it; arianna@sssup.it; paolo.dario@sssup.it).

Color versions of one or more of the figures in this paper are available online at http://ieeexplore.ieee.org.

Digital Object Identifier 10.1109/TMECH.2010.2084582 
target large production or even mass production of robots), the choice of suitable range sensors was very limited. Currently, commercial TOF laser scanners commonly used in robotics are the SICK LMS-200 [9], and the HOKUYO URG-04 LX [10]. The measurement range of TOF laser range scanners is in the order of meters [11]-[15], but these scanners require very accurate and cumbersome electronics for picosecond time resolution, which results in the use of large and costly components. Though offering much shorter distance range capabilities, triangulation laser range scanners require much simpler electronics and can be integrated in small volumes [16], [17]. Based on the aforementioned comparison, a triangulation laser scanner was finally selected, as a satisfactory compromise between size, complexity, cost, functionalities, power consumption, and integration into miniature devices. With the advance in technology, the basic components of a triangulation laser scanner, i.e., laser emitter and camera, become smaller and less power demanding; therefore, it is already possible to envision the integration of triangulation laser scanners on board centimeter-sized microrobots.

This paper is organized as follows. Section II describes the measuring principle and equations for designing a triangulation laser scanner. Section III presents the experimental setup, including hardware and software, the experimental results of the device functionalities, as well as a preliminary characterization. Finally, the concluding remarks are given in Section IV.

\section{Design of a Triangulation Laser ScAnNer}

The triangulation laser scanner consists of a laser source and at least one camera. When the laser beam emitted by the laser source strikes a detected object, the scattered beam is focused on the image sensor by means of a suitable lens. Depending on how far away the laser beam strikes a surface, the laser dot appears at different places in the camera's field of view.

\section{A. Configurations of Laser and Camera on Miniature Robots}

The triangulation laser scanner offers different features, depending on how the laser and camera are integrated in the robot. The following functionalities can be implemented:

1) the capability of detecting objects in order to avoid them;

2) the capability of measuring distances to objects;

3) the capability of scanning for holes in the floor;

4) the capability of evaluating geometric features of an object, e.g., height and width. This aspect could be of interest, for example, in the self-assembly of modular robots, in order to decide how many robots need to be gathered to form an assembled robotic organism that is capable of crossing over the obstacle.

The position of the camera and of the laser must observe strict integration constraints, which could limit performance. In the miniature Replicator robot, which has docking units on each side except for the top and bottom, the camera and laser diode are mounted on both the front and rear sides, providing two configurations.

1) Configuration 1: On the front side, the laser is placed under the docking unit with a small positive inclination, while the camera is placed above the docking unit with a level optical axis. This configuration, as shown in Fig. 1(a), allows the scanner to detect high and far objects; The sketch of configuration 1 is shown in Fig. 1(c).

2) Configuration 2: A configuration that is complementary to configuration 1 is mounted on the rear side [see Fig. 1(b)]. The laser is mounted above the docking unit with a small negative inclination, while the camera is placed under the docking unit with a level optical axis. This configuration allows the scanner to detect short objects and holes in the floor. The sketch of configuration 2

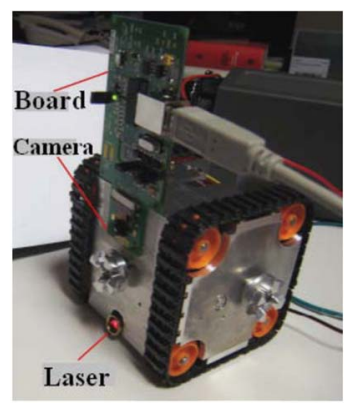

(a)

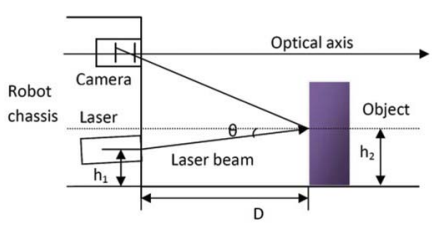

(c)

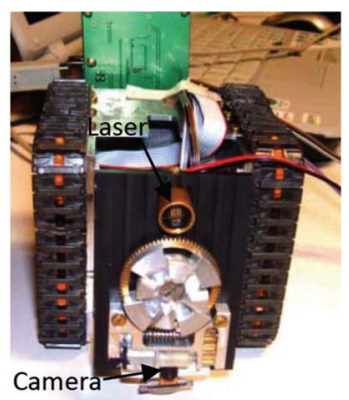

(b)

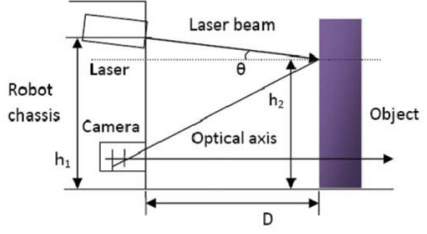

(d)
Fig. 1. Configurations of the triangulation laser scanner on the Replicator robot. (a) Laser and camera on front side. (b) Laser and camera on rear side. (c) Sketch of configuration for laser tilting up small angle on front side. (d) Sketch of configuration for laser tilting down small angle on rear side.

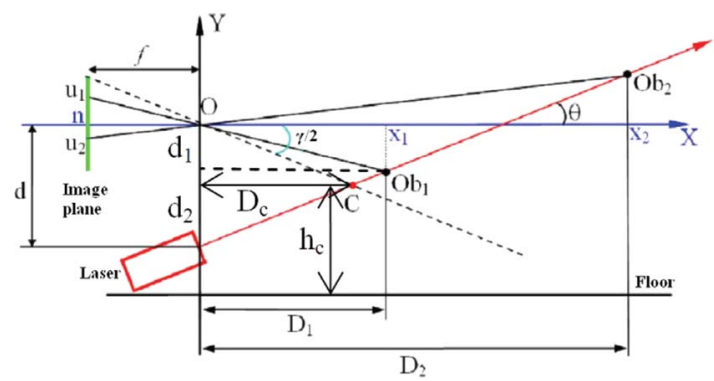

Fig. 2. Distance calculation on the basis of the known parameters $f, d$, and $\theta$. $O$ is the optical center of the camera lens.

is shown as Fig. 1(d). In addition, the bending degree of freedom of the rear side of the robot can be actuated in order to provide the laser scanner with a controlled and monitored movement, and a continuous laser sweep of the entire object, thus enabling 3-D object detection.

\section{B. Formulas for Measuring Distance, Width, and Height of Objects}

Normally, the scanner is calibrated before carrying out the measurement. When device parameters are unknown, a calibration technique is introduced [18]. In some cases, the laser-camera distance $d$ and angle $\theta$ of the laser beam over the optical axis of the camera are known, as sketched in Fig. 2; thus, distance $D$ can be calculated using (4) without the need for any calibration process

$$
\begin{aligned}
d_{1} & =\frac{u D}{f} \\
d_{2} & =D \tan \theta \\
d & =d_{1}+d_{2} \\
D & =\frac{f d}{f \tan \theta+u}
\end{aligned}
$$




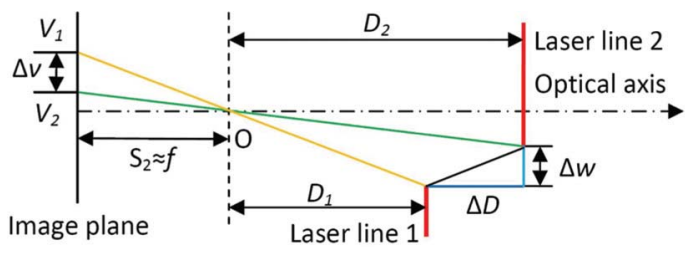

Fig. 3. Sketch of distance between end points of two laser lines.

where $f$ is the focal length of the camera, $d$ is the distance from the optical center of the camera to the laser source, $u$ is the vertical distance from the position, where the reflected laser beam strikes the image plane to the middle line of the image plane.

Fig. 3 schematically represents the acquisition of the laser light in two different positions, $u_{1}$ and $u_{2}$, on the image plane. Horizontal distance $\Delta w$ can be calculated using the following equation:

$$
\Delta w=\left|\frac{v_{1} D_{1}-v_{2} D 2}{S_{2}}\right|
$$

where $v_{1}$ and $v_{2}$ are the horizontal distances from the centroid of the reflected laser dots in the image plane to the middle of the image plane, $D$ is the distance from the camera to the object, $S_{2}$ is the distance from the optical center of the camera to the image plane, $S_{2} \approx f$ when distance $D$ is large enough. Under different situations, $\Delta w$ can be considered as the width of an object, a hole, or a gateway when the laser beam is projected on such elements.

Height $h_{2}$ from the laser spot, which strikes the detected object, to the ground, shown in Fig. 1(c) and (d), can be calculated by the following equation:

$$
h_{2}=h_{1}+D \tan \theta
$$

where $h_{1}$ is the height from the laser source to the ground, $h_{2}$ is the height from the laser dot on the detected object to the ground, $\theta$ is the laser beam inclination angle, with a positive or negative $\theta$ indicating whether the laser tilts up or down, respectively.

\section{Theoretical Measurement Range and Resolution}

Considering a more general condition $(\theta \neq \gamma / 2)$, as shown in Fig. 2, lower limit $D_{\mathrm{m} \text { in }}$ of the measurement range is due to intersection $C$ between the laser beam and the angle of view of the camera and is given by the $x$-coordinate of intersection $C$

$$
D_{\text {min }}=D_{c}=d \frac{c \tan (\gamma / 2)}{1+\tan \theta \cdot c \tan (\gamma / 2)} .
$$

The absolute value of the derivative of (4) with respect to $u$ is as follows:

$$
\left|\frac{d D}{d u}\right|=\left|\frac{D^{2}}{f d}\right| .
$$

This equation reveals a common limiting factor in triangulation systems: resolution $\left((d D / d u)^{-1}\right)$ decreases with an increase in measurement distance. In addition, knowing that the camera's focal length is $2.56 \mathrm{~mm}$ and that pixel size is $640 \times 480$, it is also possible to estimate the resolution for all possible distances $D$ from the scanner to the objects, while tuning the laser-camera distance $d$. It also follows that the achievable resolution at a specific distance $D$ is the same between system configurations with same $d$ and different tilted laser (different $\theta$ ): this is evidently true only in the common distance measurement range, while the average resolutions for the whole measurement ranges of the diverse configurations are different, i.e., higher average resolution for higher tilting angles.

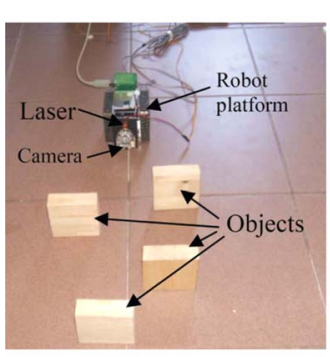

(a)

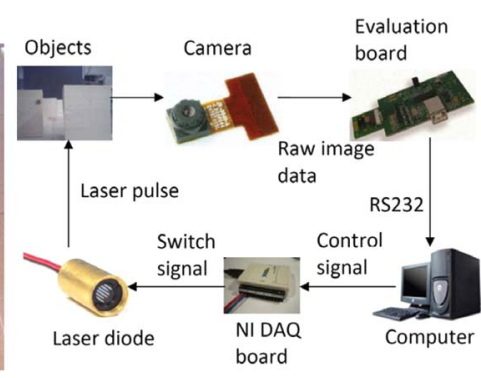

(b)
Fig. 4. (a) Experimental setup. (b) Hardware architecture and data flow.

By comparing (7) and (8), it can be deduced that both $D_{\min }$ and resolution depend on $d$, but in an opposite way. As $d$ increases, both resolution and minimum measurable distance $D_{\mathrm{min}}$ can be increased; instead, as $d$ decreases, shorter distance can be measured, but at the expense of a worse resolution.

In order to arrive at a reasonable choice, another factor needs to be considered: the minimum height of objects that the robot was to detect (see Fig. 2). This height corresponds to the height of intersection $C$ over the floor (see bottom line in Fig. 2), which can be calculated by

$$
H_{\mathrm{min}}=h_{c}=d \frac{\tan \theta \cdot c \tan (\gamma / 2)}{1+\tan \theta \cdot c \tan (\gamma / 2)}+h_{1}
$$

where $h_{1}$ is the height of the laser exit window on the floor [see Fig. 1 (c)]. After finding the intersection of the two curves $H_{\text {min }}$ and $D_{\text {min }}$, it is straightforward to demonstrate that angle $\theta$, which allows the best minimum compromise to be reached for the parameters, is $45^{\circ}$, independent of $d$.

With regard to the target applications of safe navigation, it is more important for the robot to detect a low-height object, rather than to detect an object at short distance. This means it is better to find a solution that is more suitable for correct $H_{\mathrm{min}}$ rather than for $D_{\min }$. Furthermore, focusing the laser line too far is not plausible, because it will become too weak to be seen by the camera.

Since there were no specifications as to robot resolution, it was decided that the system should be preliminarily set to make the detection between robots at a distance of about $600 \mathrm{~mm}$, the laser-camera distance was set to $40 \mathrm{~mm}$, which well suited the mechanical constraints in the current robot design. This means that at a distance of $600 \mathrm{~mm}$, the laser line strikes an object at a height of $75 \mathrm{~mm}$ over the laser (robot height is $100 \mathrm{~mm}$, minus $h_{1}$, that is, $10 \mathrm{~mm}$ ). It follows that the laser tilting angle $\theta$ is about $7^{\circ}$. Consequently, $H_{\mathrm{min}}=20.5 \mathrm{~mm}$ and $D_{\text {min }}=85.5 \mathrm{~mm}$, which are reasonable values. In the range of $D_{\text {min }}$ $-600 \mathrm{~mm}$, according to (8) and considering the limit of 1 pixel resolution, resolution ranges from less than $1 \mathrm{~mm}$ up to about $12 \mathrm{~mm}$, which are also acceptable values for generic robot exploration applications. If for any specific application requirement, this value should be changed, it will be possible to slightly tune $d$ and $\theta$.

In general, the optimal design should aim at a compromise between spatial resolution requirements, minimum detectable distance, and minimum object height.

\section{EXPERIMENTAL SETUP AND RESUlTS}

\section{A. Experimental Setup Hardware}

The current experimental setup consists of a miniature robot platform, a laser diode, a camera, several dummy objects (small blocks of wood), as shown in Fig. 4(a), and a personal computer, which interfaces with the robot. 
1) Miniature robot platform: The miniature Replicator robot platform is a tracked wheel mobile robot. Its overall size is $100 \times$ $100 \times 100 \mathrm{~mm}^{3}$. The robot can move freely on flat floors or cross small obstacles (about $6 \mathrm{~mm}$ in height).

2) Laser diode: A laser-line generator (LT-1-650-CL02 from Electron Ltd., Taiwan, China) was used. The laser works at $650 \mathrm{~nm}$ wavelength (visible red) and with a settable power. The choice of this wavelength is due to the fact that it assures better camera response and is easier to debug and calibrate than IR wavelengths. The laser was customized with a diffractive optical lens with a fan angle of $58^{\circ}$, thus occupying all the camera scene, since the camera's horizontal angle of view is about $50^{\circ}$. Currently, the laser was manufactured to generate a laser line with a focus around $200 \mathrm{~mm}$; the laser line is around $4 \mathrm{~mm}$ thick at such a distance. The laser diode is $22 \mathrm{~mm}$ long and has a diameter of $10.5 \mathrm{~mm}$. To make the laser eye-safe, a power output of $1 \mathrm{~mW}$ was set; consequently, this makes the device a class 2 product according to the laser safety classification [19], [20].

3) Camera: A miniaturized camera OV7670 from Omnivision with a package size of $6 \times 6 \times 4.8 \mathrm{~mm}$ was used for robot's vision system, which includes a CMOS image sensor with $640 \times 480$ pixel resolution and a lens with a focal length of $2.56 \mathrm{~mm}$. The image plane area is $2.36 \times 1.36 \mathrm{~mm}$. A camera evaluation board OV7670 EFXA is currently used to interface the camera to a personal computer because the embedded camera circuit for the final Replicator robot is under development.

Fig. 4(b) shows the data flow for data acquisition and processing by the hardware. The laser is controlled by the computer via a NI DAQ board from National Instruments, while raw-image data are transmitted from the evaluation board to the computer via universal serial bus. The sum of power consumption of the laser diode and the camera is less than $20 \mathrm{~mW}$. At the moment, each LT-1-650 laser diode costs around 30; therefore, the triangulation laser scanner is far cheaper than the TOF laser scanner, which normally costs several thousands of euros.

\section{B. Software}

Control of the laser diode, image acquisition and further processing are performed by a software developed in LabView from National Instruments. The general data-processing steps include image acquisition, image processing, and data postprocessing. First, camera captures images including detected objects and projected laser lines; then, image-processing algorithms are applied to extract the laser lines from the image background; finally, the aforementioned formulas are applied to calculate the distance, width, and height of the projected laser lines.

1) Laser lines extraction: Image-processing algorithms, e.g., Gaussian filtering, are applied during the image-processing stage to condition the images for more effective background removal, then color value or gray-scale threshold, is applied to extract the laser lines from the image background.

2) Particles filtering: Since small and short light dots/lines in the image plane are considered as undesired particles compared to longer and larger light lines, the threshold values of width, length, and area are set so as to eliminate them.

3) Data post-processing: Finally, the processed images of the laser lines (see Section III-D) are stored in binary format, which needs smaller memory. These laser lines are considered as particles for computing discrete point coordinates, centroid, angle, coordinates of boundary box, etc. A transformation matrix can also be used to build the points of the projected laser lines in a real-world model. (a)

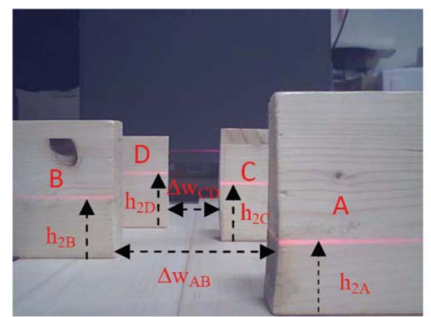

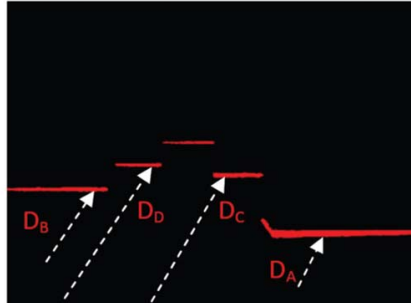

(b)
Fig. 5. Distance and gateway measurement. (a) Detected objects in front of laser scanner. (b) Segmented laser lines in binary image.

\section{Calibration}

Before conducting measuring tests, a simple calibration technique [18] may be performed, if focal length $f$ of the camera is known. First, a calibration object is placed at a distance of $80 \mathrm{~mm}$ in order to determine the value of $u$, which represents the vertical distance from the position where the reflected laser beam strikes the image plane to the middle point of the image plane; then, the same object is placed at a distance of $400 \mathrm{~mm}$, in order to determine the new value of $u$. Although the calibration process can be avoided by storing the geometrical parameters of the sensor, possible deviation can occur due to the mechanical setup; thus, the calibration process always assures more accurate measurements.

\section{Experimental Results}

Based on the aforedescribed setup, 2-D static scanning experiments were performed with respect to the two configurations (see Fig. 1). The tests reported in the following were repeated 20 times.

1) Distance measurement: The laser scanner on the rear side of the Replicator robot was tested first. The laser diode was tilted down with a angle of $7^{\circ}$, and potential objects $\mathrm{A}, \mathrm{B}, \mathrm{C}$, and D were placed in front of the laser scanner. After image acquisition and processing of the raw image, as illustrated in Fig. 5(a), the extracted binary image of the laser lines was obtained as shown in Fig. 5(b). The experiments showed that by moving the position of the objects, measurable distance range was from 80 to $600 \mathrm{~mm}$ with 1-20 mm error and 1-10 mm resolution. When distance was shorter than $80 \mathrm{~mm}$, the laser line was out of camera's view field; therefore, the laser lines could not be reflected to the image plane of the camera. Theoretically, the maximal measurable distance can be infinite; however, when distance is longer than $600 \mathrm{~mm}$, the reflected laser line on the image plane is too weak to be separated from the background with current algorithms. The scanner can detect several objects at the same time; the measuring results of distances $D_{A}, D_{B}, D_{C}$, and $D_{D}$ listed in Table I indicate that shorter the distance is, smaller the error is.

2) Width measurement: Equation (5) was used for calculating the horizontal width $\Delta w$ between two objects that constitute a gateway. The measuring results of width $\Delta w_{A B}$ and $\Delta w_{C D}$ are listed in Table I. Hole width can also be detected by using the same principle employed for detecting the gateway.

3) Height measurement: Laser altitude height $h_{1}$ was $70 \mathrm{~mm}$, when mounted on the rear side of the Replicator robot, and altitude height $h_{2}$ of the laser line on the detected objects is calculated automatically using equation (6). The measuring results of $h_{2}$ are listed in Table I.

4) Angle measurement: The surrounding environment can also be interpreted by measuring the angle of laser lines relative to the 
TABLE I

MEAsuring Results of 2-D STATIC SCANNING

\begin{tabular}{|c|c|c|}
\hline Items & measuring result & error \\
\hline$D_{\min }(\mathrm{mm})$ & 80 & 1 \\
\hline$D_{\max }(\mathrm{mm})$ & 600 & 20 \\
\hline$D_{A}(\mathrm{~mm})$ & 101 & 1 \\
\hline$D_{B}(\mathrm{~mm})$ & 122 & 2 \\
\hline$D_{C}(\mathrm{~mm})$ & 196 & 3 \\
\hline$D_{D}(\mathrm{~mm})$ & 390 & 6 \\
\hline$\Delta W_{A B}(m m)$ & 35 & 3 \\
\hline$\Delta W_{C D}(m m)$ & 10 & 2 \\
\hline$h_{2 A}(\mathrm{~mm})$ & 15 & 2 \\
\hline$h_{2 B}(\mathrm{~mm})$ & 18 & 2.5 \\
\hline$h_{2 C}(\mathrm{~mm})$ & 23 & 2.5 \\
\hline$h_{2 D}(\mathrm{~mm})$ & 30 & 3 \\
\hline$\theta_{1}($ degree $)$ & 10 & 2 \\
\hline$\theta_{2}($ degree $)$ & 30 & 3 \\
\hline
\end{tabular}

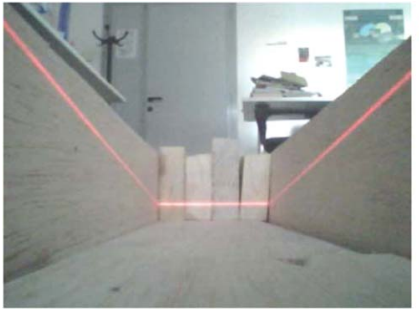

(a)

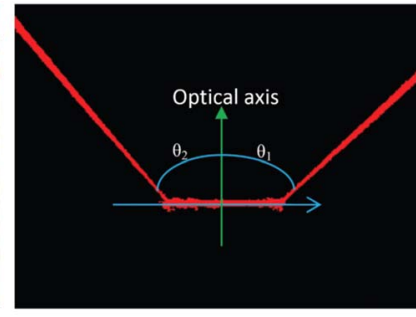

(b)
Fig. 6. Angle measurement by laser range scanner. (a) Acquired raw image including laser lines. (b) Processed binary image only including laser lines.

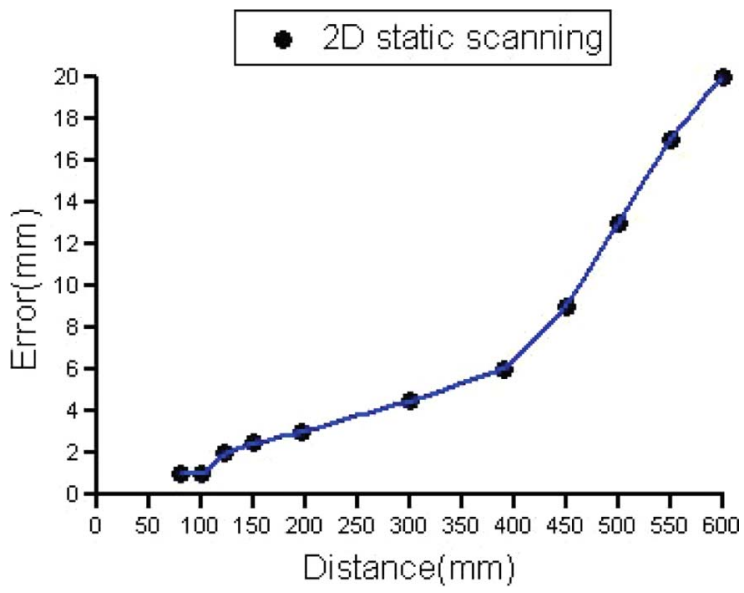

Fig. 7. Relationship between distance and measuring error.

camera's optical axis. The measuring results of angles $\theta_{1}$ and $\theta_{2}$ indicated in Fig. 6(b) are listed in Table I and can be applied to corridor recognition and analysis. The relationship between distance and measuring error is shown in Fig. 7. Due to the same absolute value of the laser tilting angle, configuration 1 [see Fig. 1(a)] was changed to configuration 2 [see Fig. 1(b)] after rotating acquired images by $180^{\circ}$.

\section{E. Preliminary Characterization}

Preliminary tests were conducted to understand how performances of the scanner were affected by ambient light, by the incidence angle of the laser beam, and by the objects' surface properties (color and reflectivity).
1) Ambient light: During experiments, it was observed that ambient light strongly influenced the measuring results. When the indoor environment was dark, the laser lines were reflected to the image sensor very clearly and were easy to be separated from the background. When the range scanner worked under strong sunlight, the laser lines on the acquired image were weak with respect to the background; as a result, it was difficult to segment them from the background, if $D$ was larger than $600 \mathrm{~mm}$.

2) Incidence angle of the laser beam for configuration 2: When the tilting angle of the laser beam was increased, a sharp laser line appeared on detected objects, whereas it becomes diffractive with a smaller tilting angle. The results show laser beam has a large incidence angle, the measuring results are more robust and more accurate.

3) Properties of the detected object: As known, object reflectivity is based upon the object's material and color. Thus, surface reflectivity influences the intensity of the reflected laser line on the image plane. The experiments indicated that the reflected laser line was weakest when black objects were scanned under standard light conditions during daytime. In these cases, the maximal measurable distance dropped to $200 \mathrm{~mm}$.

The experimental tests confirmed good performance and reliability of the laser scanner that was able to measure distance up to $600 \mathrm{~mm}$ with high accuracy; however, several disadvantages have to be addressed as detailed in the following.

Although there are several known ways to reduce the interference of environmental light, this remains a limiting factor for the system performance. Currently, the software performs the image subtraction between the two following acquired images: image $\mathrm{A}$, which is taken with the laser switched ON, and image B with the laser switched OFF. The difference between the two images $(A-B)$ gives an image that theoretically consists of the laser lines only [21], [22]. The technique performs well when the environment light is not too strong to cause saturation of the camera pixels (in this case, the laser's added light intensity would evidently be lost) and when the background image is stable in terms of both illumination and scene in the time frame of image $\mathrm{A}$ and $\mathrm{B}$ acquisition. Noise can remain following the difference between image A and B, but it can be easily filtered by thresholds or erosion-like algorithms. If the robot has to perform in an area where there are other robots moving, the speed of acquisition of the two images and its synchronization with the laser operation are important factors in order to maintain the same scene. Considering that the camera could reach a maximum speed of 30 frames per second, and considering that the robot moves at a speed of $20 \mathrm{~mm} / \mathrm{s}$, the scene will be slightly changed in the two frames and further image processing will probably be necessary. From hardware viewpoint, a narrow bandpass filter could also be an effective solution for eliminating the interference of ambient light: a $650 \mathrm{~nm}$ wavelength filter, for example, would permit only the red light to be transmitted to the camera. However, this solution would limit the capabilities of the camera as a robotic vision tool.

\section{CONCLUSION AND Future WORK}

A miniature triangulation laser range scanner, suitable for integration into miniature mobile robots used for robotic swarms or modular robotic self-assembly, was designed, developed, and characterized. The scanner demonstrates satisfactory performance both in terms of measurement range, resolution, and accuracy, and it offers useful capabilities for the detection of the geometrical features of objects, which can be exploited for navigation purposes and for an improved robot awareness of the surrounding environment. Future work will focus on improving image-processing algorithms and in pursuing active laser scanning by 
rotating the whole laser-camera system mounted on a movable side of the robot; furthermore, cooperative 3-D laser scanning systems implemented by swarm robots or self-assembling modular robots will be investigated.

\section{ACKNOWLEDGMENT}

The authors would like to thank L. Ricotti and N. Funaro for fabricating prototypes of the Replicator robotic mechanical platform. The authors are also grateful to all the members involved in the Replicator project for their helpful suggestions and discussions.

\section{REFERENCES}

[1] T. Dutta and G. Fernie, "Utilization of ultrasound sensors for anti-Collision systems of powered wheelchairs," IEEE Trans. Neural Syst. Rehabil. Eng., vol. 13, no. 1, pp. 24-32, Mar. 2005.

[2] F. Abrate, M. Indri, and B. Bona, "Monte carlo localization of minirovers with low-cost IR sensors," in Proc. IEEE/ASME Int. Conf. Adv. Intell. Mechatron. (AIM), ETH Zurich, Switzerland, 2007, pp. 1-6.

[3] H. H. Cheng, B. D. Shaw, J. Palen, J. E. Larson, X. D. Hu, and K. Van, "A real-time laser-based detection system for measurement of delineations of moving vehicles," IEEE/ASME Trans. Mechatronics, vol. 6, no. 2, pp. 170-187, Jun. 2001.

[4] O. Duran, K. Althoefer, and L. D. Seneviratne, "Pipe inspection using a laser-based transducer and automated analysis Techniques," IEEE/ASME Trans. Mechatronics, vol. 8, no. 3, pp. 401-409, Sep. 2003.

[5] J. C. Zufferey and D. Floreano, "Toward 30-gram autonomous indoor aircraft: vision-based obstacle avoidance and altitude control," in Proc. 2005 IEEE Int. Conf. Robot. Autom., Barcelona, Spain, 2005, pp. 2594 2599.

[6] H. Suzuki and M. Minami, "Visual servoing to catch fish using global/local GA search," IEEE/ASME Trans. Mechatronics, vol. 10, no. 3, pp. 352357, Jun. 2005.

[7] E. C. Dean-Leon, V. Parra-Vega, and A. Espinosa-Romero, "Visual servoing for constrained planar robots subject to complex friction," IEEE/ASME Trans. Mechatronics, vol. 11, no. 4, pp. 389-400, Aug. 2006.

[8] REPLICATOR. [Online]. Available: http://www.replicators.eu/ (Accessed in 2010).

[9] SICK. [Online]. Available: http://www.sick.com/group/EN/ (Accessed in 2010).

[10] HOKUYO. [Online]. Available: http://www.hokuyo-aut.jp/ (Accessed in 2010).

[11] C. Ye and J. Borenstein, "A novel filter for terrain mapping with laser rangefinders," IEEE Trans. Robot. Autom., vol. 20, no. 5, pp. 913-921, Oct. 2004.

[12] D. M. Cole and P. M. Newman, "Using laser range data for 3D SLAM in outdoor environments," in Proc. IEEE Int. Conf. Robot. Autom., Orlando, FL, May 2006, pp. 1556-1563.

[13] V. Nguyen, A. Harati, A. Martinelli, R. Siegwart, and N. Tomatis, "Orthogonal slam: A step toward lightweight indoor autonomous navigation," in Proc. IEEE/RSJ Int. Conf. Intell. Robots Syst., Beijing, China, 2006, pp. 5007-5012.

[14] S. Grzonka, G. Grisetti, and W. Burgard, "Towards a navigation system for autonomous indoor flying," in Proc. IEEE Int. Conf. Robot. Autom., Kobe, Japan, 2009, pp. 2878-2883.

[15] E. Asadi and M. Bozorg, "A decentralized architecture for simultaneous localization and mapping," IEEE/ASME Trans. Mechatronics, vol. 14, no. 1, pp. 64-71, Feb. 2009.

[16] K. Yoshida and S. Hirose, "Laser triangulation range finder available under direct sunlight," in Proc. Int. Conf. Robot. Autom., 1998, vol. 3 , pp. $1702-1708$.

[17] K. Konolige, J. Augenbraun, N. Donaldson, C. Fiebig, and P. Shah, "A low-cost laser distance sensor," in Proc. Int. Conf. Robot. Autom., 2008, pp. 3002-3008.

[18] H. G. Nguyen and M. R. Blackburn, "A simple method for range finding via laser triangulation," Naval Command, Control and Ocean Surveillance Center, San Diego, CA, Tech. Rep., 1995.

[19] Laser Safety: Safety of Laser Products-Part 1: Equipment Classification and Requirements, 2nd ed., IEC, Geneva, Switzerland, 2007.

[20] American National Standard for the Safe Use of Lasers, ANSI Standard 2136.1-1993, p. 31, 32, 41, 42, 1993.
[21] M. Seelinger, "Development of a semi-autonomous multi-task robotics package using camera-space manipulation," Ph.D. dissertation, Univ. Notre Dame, Notre Dame, IN, 1996, pp. 51-61.

[22] B. Zhang, "Three-dimensional laser-assisted image analysis for robotic surface operation with camera-space manipulation," Ph.D. dissertation, Univ. Notre Dame, Notre Dame, IN, 2007, pp. 29-30.

\section{Command State-Based Modifiable Walking Pattern Generation on an Inclined Plane in Pitch and Roll Directions for Humanoid Robots}

\author{
Young-Dae Hong, Bum-Joo Lee, and Jong-Hwan Kim
}

\begin{abstract}
Previous research related to walking on an inclined plane for humanoid robots, including the 3-D linear inverted pendulum model (3DLIPM) approach, were unable to modify walking period, step length, and walking direction independently without any additional step for adjusting the center of mass (CoM) motion. Moreover, the inclination along the pitch direction was only considered for walking. To solve these problems, a novel command state (CS)-based modifiable walking pattern generator for humanoid robots is proposed for modifiable walking on an inclined plane in both pitch and roll directions. The dynamic equation of the 3D-LIPM on the inclined plane in both pitch and roll directions is derived to obtain the CoM motion. Using the CoM motion, a method for modifiable walking pattern generation on the inclined plane is developed to follow a given CS composed of walking periods, step lengths, and walking directions for both legs. The effectiveness of the proposed walking pattern generator is demonstrated through both simulation and experiment for the small-sized humanoid robot, HanSaRam-IX (HSR-IX).
\end{abstract}

Index Terms-3-D linear inverted pendulum model (3D-LIPM), command state (CS), humanoid robot, modifiable walking pattern generator (MWPG), walking on inclined plane, zero-moment point (ZMP).

\section{INTRODUCTION}

Research on humanoid robots has made rapid progress such that various humanoid robots capable of stable walking with control algorithms have been developed [1]-[5]. A lot of control algorithms have been presented on the assumption that the plane is flat. However, in human environments, there exist not only flat but also inclined planes. Therefore, walking pattern generation on the inclined plane for humanoid robots is one of the key research issues.

As research on walking pattern generation on the inclined plane, Kajita and Tani proposed a linear inverted pendulum model (LIPM) to

Manuscript received July 9, 2010; revised September 2, 2010; accepted October 8, 2010. Date of publication December 3, 2010; date of current version May 11, 2011. Recommended by Technical Editor A. Menciassi. This work was supported by the Basic Science Research Program through the National Research Foundation of Korea funded by the Ministry of Education, Science and Technology under Grant 2010-0000831.

The authors are with the Department of Electrical Engineering, Korea Advanced Institute of Science and Technology (KAIST), Daejeon 305-701, Korea (e-mail: ydhong@ rit.kaist.ac.kr; bjlee@ rit.kaist.ac.kr; johkim@rit.kaist.ac.kr).

This paper has supplementary downloadable material available at $\mathrm{http}: / /$ ieeexplore.ieee.org, provided by the author. This material includes two videos with a total size of $2.81 \mathrm{MB}$. Contact johkim@ @it.kaist.ac.kr for further questions about this work.

Digital Object Identifier 10.1109/TMECH.2010.2089530 